\title{
Analytic stability criteria for edge MHD oscillations in high performance ELM free tokamak regimes
}

\author{
D. Brunetti, ${ }^{1, *}$ J. P. Graves, ${ }^{2}$ E. Lazzaro, ${ }^{1}$ A. Mariani, ${ }^{2}$ S. Nowak, ${ }^{1}$ W. A. Cooper, ${ }^{2}$ and C. Wahlberg ${ }^{3}$ \\ ${ }^{1}$ Istituto di Fisica del Plasma IFP-CNR, Via R. Cozzi 53, 20125 Milano, Italy \\ ${ }^{2}$ Swiss Plasma Center (SPC), CH-1015 Lausanne, Switzerland \\ ${ }^{3}$ Department of Physics and Astronomy, P.O. Box 516, Uppsala University, SE-751 20 Uppsala, Sweden
}

(Dated: September 21, 2017)

\begin{abstract}
A new dispersion relation, and associated stability criteria, is derived for low- $n$ external kink and infernal modes, and is applied to modelling the stability properties of quiescent $\mathrm{H}$-mode like regimes. The analysis, performed in toroidal geometry with large edge pressure gradients associated with a local flattening of the safety factor, includes a pedestal, sheared toroidal rotation and a vacuum region separating the plasma from an ideal metallic wall. The external kink-infernal modes found here exhibit similarities with experimentally observed Edge Harmonic Oscillations.
\end{abstract}

\section{INTRODUCTION}

The tokamak high-confinement regime (H-mode) is usually accompanied by sudden and violent events called Edge Localised Modes (ELMs). These energy bursts are of extreme concern since high energy and particle loads are deposited on the plasma facing components which can lead to a severe deterioration of the materials and contamination of the plasma. In contrast, the quiescent high confinement regime (QH-mode) $[1,2]$ avoids ELMs but nevertheless has features of high performance such as large edge pressure gradients and high energy confinement times.

Low- $n(\sim 1)$ magnetohydrodynamic (MHD) perturbations called edge harmonic oscillations (EHOs) are always observed during the QH-mode operation [1-6]. In JET experiments a similar MHD instability called the Outer Mode closely resembles the EHO structure [7]. These instabilities replace the ELM activity (ELMs in contrast have $n \gg 1$ ) and the associated energy loads on the materials facing the plasma are much lower as compared to regimes where ELMs are present. These benign low- $n$ oscillations have been linked with kink/peeling modes (perturbations localised near the plasma edge driven by edge current and pressure gradients) [8] and have therefore been proposed as a possible candidate for the explanation of the appearance of these perturbations.

The steep edge pressure gradient in the low collisionality regime is associated with a significant bootstrap contribution to the current which causes in turn a plateau in the safety factor profile. Numerical studies of low- $n$ MHD instabilities in the $\mathrm{QH}$-mode regime with a plateau in $q$ near the edge have been found to have infernal-like features [911]. The single Fourier harmonic of these structures has a radial extension much larger than peeling modes. Three dimensional free boundary MHD equilibria simulations of JET and TCV-like plasmas with large edge bootstrap current exhibit a distorted boundary with a dominant $n=1$ Fourier component driven mainly by non axisymmetric contributions to the parallel current density [12-14]. Nonlinear simulations

\footnotetext{
${ }^{*}$ Electronic address: brunetti@ifp.cnr.it
}

of QH-mode DIII-D plasma discharges showed that medium$n(\sim 3,4,5)$ harmonics are linearly the most unstable modes but the nonlinear phase is dominated by saturated stationary low- $n$ modes [15].

Since EHOs exhibit similarities with infernal type modes, the aim of this paper is to identify and isolate the specific physical conditions for edge infernal type MHD instabilities using analytic methods. Since experimental evidence (see e.g. $[1,5]$ ) and nonlinear simulations [15] dictate that low- $n$ (especially $n=1$ ) modes are dominant, we concentrate on the linear stability properties, and marginal stability conditions, for low- $n$ ideal modes. Critical to the work is the inclusion of low magnetic shear and steep pressure gradients in the edge and the extension of magnetic perturbations across a vacuum region to a conducting wall. Although more realistic configurations should include a separatrix, our analysis is concentrated on limited geometry in line with Ref. [8]. This avoids the mathematical complications of the separatrix, still providing various features which closely resemble the experimental findings. We begin by mathematically describing the geometry and physical model, i.e. the infernal mode equations are introduced and a discussion on their validity on the applicability to our problem is given. The analysis is carried out in three separate regions. First we solve the vacuum and the internal regions. The edge region where the flattening of the safety factor occurs is then solved exactly for a particular class of pressure and mass density profiles. The dispersion relation (of a Bussac-like class [16]) is derived by matching the solutions of the three regions and then the parametric dependency for instability is investigated.

\section{PHYSICAL MODEL}

The equilibrium considers a tokamak configuration with shifted circular toroidal surfaces having a strong axial toroidal field $\left(B_{0}\right)$ and an $\varepsilon$ times smaller poloidal field $\left(B_{P}\right)$ with $\beta=p / B_{0}^{2} \sim O\left(\varepsilon^{2}\right)$ ( $p$ is the pressure having normalised $\left.\mu_{0}=1\right)$. Here $\varepsilon=a / R_{0} \ll 1$ where $R_{0}$ and $a$ are the major and minor radii respectively. We allow for toroidal rotation effects, under the assumption that the plasma rotation frequency is significantly smaller than the Alfvén fre- 
quency. We use a right handed straight field line coordinate system $(r, \vartheta, \varphi)$ where $r$ is a flux label with the dimensions of length, $\vartheta$ (counter-clockwise) and $\varphi$ are the poloidal-like and toroidal angles respectively. The contravariant basis vectors are $\nabla C^{i}$ with $C^{i}=(r, \vartheta, \varphi)$. The magnetic field in the plasma is $\boldsymbol{B}=T \nabla \varphi-\nabla \psi \times \nabla \varphi$, where $\psi$ is the poloidal flux. The safety factor profile is denoted with $q$ and we set $\iota=1 / q$.

The plasma is described by the ideal MHD equations [17]:

$$
\begin{aligned}
\rho\left[\partial_{t} \boldsymbol{v}+\boldsymbol{v} \cdot \boldsymbol{\nabla} \boldsymbol{v}\right] & =-\nabla p+\boldsymbol{J} \times \boldsymbol{B}, \\
\partial_{t} \boldsymbol{B}=\boldsymbol{\nabla} & \times(\boldsymbol{v} \times \boldsymbol{B}), \\
\partial_{t} p+\boldsymbol{v} \cdot \nabla p+\Gamma p \nabla \cdot \boldsymbol{v} & =0, \quad \partial_{t} \rho+\nabla \cdot(\rho \boldsymbol{v})=0,
\end{aligned}
$$

where $\boldsymbol{v}$ is the plasma MHD velocity, $\rho$ is mass density, $\boldsymbol{J}=\boldsymbol{\nabla} \times \boldsymbol{B}$ the current density and $\Gamma=5 / 3$ is the adiabatic index. Hereafter with $\rho_{0}$ and $p_{0}$ we denote the equilibrium mass density and pressure profiles, while $B_{0}$ and $\bar{\rho}$ are the magnetic field and the mass density on the magnetic axis. Other equilibrium quantities are denoted by the subscript 0 . Perturbed quantities have a dependence on time and angular variables of the type $\exp [i(\ell \vartheta-n \varphi)+\gamma t]$. Since $n$ is fixed, we omit to specify the toroidal mode number in writing the Fourier components. If rotation is allowed, the eigenvalue $\gamma$ can be complex. We stress the point that the plasma is considered ideal, so that the formation of tearing-like perturbations is prevented.

We assume a step-like equilibrium current density profile for which the safety factor is constant for $0<r<r_{0}$ and $r_{*}<r<a$, with values $\iota_{a x}$ and $\iota_{*}$ respectively $\left(\iota_{a x}>\iota_{*}=\right.$ $1 /(m / n-\delta q))$ while $\iota=\iota_{*}\left(r_{*} / r\right)^{2}$ in the region $r_{0}<r<r_{*}$. A basic assumption for the following analysis is that $\delta q / q \ll 1$, i.e. the flat $q$ region is nearly resonanting with $m / n$. We refer to the internal region $0<r<r_{*}$ as the inner region, while the region $r_{*}<r<a$ is the pedestal low-shear region whose middle point is $r_{p}=\left(r_{*}+a\right) / 2$. Assuming a wall of thickness $d$, the vacuum region extends from $r=a$ to $r=b$ and for $r>b+d$. The shape of $q$ and the relevant radial positions are shown in Fig. 1. We anticipate that in the next sections the analysis will consider step-like profiles for pressure, mass density and rotation, all vanishing for $r>r_{p}$.

More realistically, in a geometry with separatrix the safety factor grows up to large values. But this occurs in a region which is much narrower with respect to the region of local edge $q$ flattening, which is expected to be proportional to the radial extension of the perturbation. Thus, also motivated by Ref. [9], we expect that by choosing a safety factor of the form shown in Fig. 1 the main physical ingredients for the development of an edge MHD instability are captured (similarly to what has been presented in Refs. $[10,11])$.

Such a profile is associated with a piecewise constant current density. Although we have an edge current density, according to Ref. [18] we assume to operate inside the stability window against low- $n$ ideal external kink modes in the $\left(q_{0}, q_{a}\right)$ parameter space ( $q_{0}$ and $q_{a}$ are the values of the safety factor on the magnetic axis and at the edge respectively), i.e. requiring that $q_{a} / q_{0} \gg 1$. Finally, because a background stationary flow is assumed, we introduce the Lagrangian fluid displacement $\boldsymbol{\xi}$ related to the perturbed plasma MHD velocity $\tilde{\boldsymbol{v}}$ by the relation $\tilde{\boldsymbol{v}}=\partial_{t} \boldsymbol{\xi}+\boldsymbol{v}_{0} \cdot \nabla \boldsymbol{\xi}-\boldsymbol{\xi} \cdot \boldsymbol{\nabla} \boldsymbol{v}_{0}[19]$.

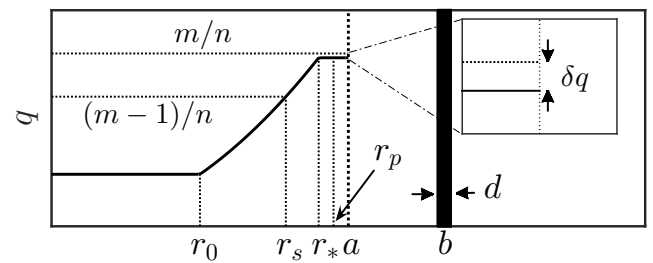

Figure 1. Shape of the safety factor employed in our model. The relevant radial positions are highlighted.

In the inner region mode coupling is absent because of the large parallel wave vector and the absence of strong pressure gradients. In this region we also neglect inertial effects. The equation describing the radial structure of $\ell$ th Fourier component of the perturbed fluid displacement $X_{\ell}=\boldsymbol{\xi}_{\ell} \cdot \nabla r$ is the Newcomb's equation $[20,21]$ :

$$
\left[r^{3} k_{\|, \ell}^{2} X_{\ell}^{\prime}\right]^{\prime}-r\left(\ell^{2}-1\right) k_{\|, \ell}^{2} X_{\ell}=0
$$

where $k_{\|, \ell}=\ell \iota-n$ and ${ }^{\prime} \equiv d / d r$.

In the pedestal low-shear region (where $q=m / n-\delta q$ ) mode coupling is allowed due to toroidicity and the combined effect of large pressure gradients and field line bending weakening [22, 23]. For a fixed toroidal number $n$, the coupling is induced by the metric oscillation in the Jacobian of the system and it involves a main mode $X_{m}$ and its two neighbouring harmonics $X_{m \pm 1}$ with $X_{m \pm 1} / X_{m} \sim \varepsilon$. Higher harmonics are assumed vanishing. In this region we allow for inertial effects assuming $\omega \sim \Omega \sim \varepsilon \omega_{A}$ where $\omega=i \gamma, \omega_{A}=B_{0} /\left(R_{0} \sqrt{\bar{\rho}}\right)$ and $\Omega$ is the plasma rotation frequency. It is also assumed that $\delta q / q \sim \omega / \omega_{A}$ with $\delta q / q \sim \varepsilon \ll 1$. The perturbations are then described by the coupled equations as in Refs. [22, 24-26]:

$$
\begin{gathered}
{\left[r^{3} Q X_{m}^{\prime}\right]^{\prime}+r\left[\left(1-m^{2}\right) Q+r\left(\frac{A_{2}}{n^{2}}\right)^{\prime}-\frac{\tilde{\alpha}^{2}}{2}+\frac{\tilde{\alpha} r}{R_{0}}\left(\frac{1}{q^{2}}-1\right)\right] X_{m}} \\
+\frac{\tilde{\alpha}}{2}\left[\frac{r^{-m}}{m+1}\left(r^{2+m} X_{m+1}\right)^{\prime}-\frac{r^{m}}{m-1}\left(r^{2-m} X_{m-1}\right)^{\prime}\right]=0 \\
{\left[r^{-1 \mp 2 m}\left(r^{2 \pm m} X_{m \pm 1}\right)^{\prime}\right]^{\prime}=\frac{1 \pm m}{2}\left[\tilde{\alpha} r^{\mp m} X_{m}\right]^{\prime}}
\end{gathered}
$$

with $Q=(\delta q / q)^{2}+A_{1} / n^{2}, \tilde{\alpha}=-\left(2 R_{0} \tilde{p}_{0}^{\prime} q^{2}\right) / B_{0}^{2}$ where $\tilde{p}_{0}=p_{0}+\rho_{0}\left(\Omega R_{0}\right)^{2} / 2$ [27] and the expressions for the inertial corrections $A_{1}$ and $A_{2}$ are given by Eqs. (6a) and (6b) in Ref. [25]. In the limit $\mathcal{M}^{2}=\left(\Omega / \omega_{A}\right)^{2} / \beta \ll 1(\mathcal{M}$ is the Mach number) and assuming that the ratio $p_{0} / \rho_{0}$ is constant, we approximate $A_{1} \simeq-\left(\rho_{0} \omega_{D}^{2}\right) /\left(\bar{\rho} \omega_{A}^{2}\right)\left[1+2 q^{2}\right]$ and $A_{2} \simeq-\left(\rho_{0} \omega / \bar{\rho} \omega_{A}^{2}\right)\left[\omega\left(1+2 q^{2}\right)+2 n \Omega\right]$ where $\omega_{D}=\omega+n \Omega$. Allowing equilibrium gradients (for $p_{0}, \rho_{0}$ and $\Omega$ ) to be large in a narrow region, we can employ Eqs. (2) and (3) under the assumption that $m \Delta \sim \varepsilon$ where $\Delta$ is the radial extension of the pedestal low-shear region. The eigenvalue equation is obtained by matching the solutions of the pedestal low-shear region across the boundary points (i.e. requiring continuity for the main harmonic and for the logarithmic derivatives of the sidebands). 


\section{VACUUM REGION}

The magnetic field in the vacuum region is written as $\boldsymbol{B}_{v}=$ $-\nabla \chi$ with the constraint $\nabla^{2} \chi=0$ [28]. The condition at the perfectly conducting metallic wall is $\hat{\boldsymbol{n}} \cdot \boldsymbol{B}_{v}=0$ [28-30], where $\hat{\boldsymbol{n}}$ is the normal vector pointing outward from the $r=a$ surface $(\hat{\boldsymbol{n}} \equiv \nabla r)$. In large aspect ratio and under the assumption $\ell>n$ for each Fourier harmonic $(\ell, n)$, the $\ell$ th component of the vacuum perturbation which fulfils the boundary condition at the metallic wall is $\chi_{\ell}(r) \sim(r / b)^{\ell}+(b / r)^{\ell}$ [28]. At the plasma-vacuum interface we have $\llbracket \hat{\boldsymbol{n}} \cdot \boldsymbol{B} \rrbracket_{a}=0$ where $\mathbb{I} \cdot \mathbb{1}_{r}=(\cdot)_{r+x}-(\cdot)_{r-x}$ with $x \rightarrow 0[29,30]$. Hereafter we shall consider $x$ as an infinitesimally small quantity. By means of the Faraday-Ohm's law we can extend the definition of perturbed displacement $X$ outside the plasma, i.e. we write $\widetilde{B}^{r}$ in terms of $X$. This yields $\tilde{B}_{\ell}^{r} \sim k_{\|, \ell} X_{\ell}$, so that since $\tilde{B}_{\ell}^{r}$ is continuous, $X_{\ell}$ also is continuous ( $k_{\|, \ell}$ is continuous by hypothesis). Thus for the Fourier mode $\ell=m \pm 1$ we have [21, 28]:

$$
\left.\frac{r X_{\ell}^{\prime}}{X_{\ell}}\right|_{a+x}=\frac{2 \iota_{*}}{\iota_{*}-n / \ell}-\frac{\ell+1+(\ell-1)(a / b)^{2 \ell}}{1-(a / b)^{2 \ell}},
$$

where in the vacuum $\iota=\iota_{*} a^{2} / r^{2}$ [21]. Concerning the Fourier mode $m$, we note that in the vacuum region having introduced the quantity $X_{m}$, the equation describing the perturbation is written in a form similar to (1). Thus we multiply (1) by $X_{m}$ and integrate by parts from $a$ to $b$ yielding:

$\left.r^{3} k_{\|, m}^{2} X_{m} \frac{d X_{m}}{d r}\right|_{a} ^{b}-\int_{a}^{b} d r\left[r^{3} k_{\|, m}^{2}\left|\frac{d X_{m}}{d r}\right|^{2}+r\left(m^{2}-1\right) k_{\|, m}^{2}\left|X_{m}\right|^{2}\right]=0$

Under the constraint $k_{\|, m}^{2}(a)=(n \delta q / q)^{2} \ll 1$ and $X_{m}(b)=0$, we are left with an integral of positive terms which must be vanishing. This automatically gives $X_{m}=0$. We shall still approximate $X_{m}(a) \approx 0$ if the resonance $q=m / n$ is in the vacuum gap at position $r_{m}$, if $\left(r_{m}-a\right) /\left(a-r_{*}\right) \ll 1$ (i.e. the distance of the resonance is much smaller than the radial width of the $q$-plateau). This is indeed the case if we allow $\delta q$ to be small, i.e. the variations in the edge safety factor are small. Note that for a mode $\ell \neq m$ for which $k_{\|, \ell}^{2}(a) \sim O(1)$ this arguments does not hold.

\section{INNER REGION}

In the region $0<r<r_{*}$ mode coupling is prevented so that different Fourier harmonics behave independently according to Eq. (1). Note that $X_{\ell}$ is smooth at $r_{0}$, as readily seen by integrating (1) across $r_{0}$. Focusing on the Fourier mode with poloidal mode number $m$, Eq. (1) is multiplied by $X_{m}$ and integrated from 0 to $r_{*}$ [17]. This produces an equation similar to (5) where we must perform the substitutions $a \rightarrow 0$ and $b \rightarrow r_{*}$. With the hypothesis $k_{\|, m}\left(r_{*}\right) \ll 1$ and applying the boundary condition $X_{m}(0)<\infty$ we have at leading order $X_{m}=0$. Equivalently one can use the $\delta W$ approach [22]. Invoking similar arguments considered for the vacuum region, we approximate $X_{m} \approx 0$ also when the resonant point $r=r_{m}$ at which $q=m / n$ is in the inner region under the condition that $\left(r_{*}-r_{m}\right) /\left(a-r_{*}\right) \ll 1$, i.e. $\delta q$ sufficiently small. For the upper $m_{+}=m+1$ mode $\left(X_{+}=X_{m+1}\right)$ the eigensolution for $r>r_{0}$ which smoothly joins to the solution obtained in region $0<r<r_{0}$ is:

$$
X_{+} \propto \frac{1}{k_{\|, m_{+}}}\left[\left(\frac{m}{m_{*}}-\left(\frac{r_{0}}{r_{*}}\right)^{2}\right)\left(r / r_{0}\right)^{m}+\frac{1}{m_{*}}\left(r / r_{0}\right)^{-m-2}\right],
$$

where $m_{*}=m-n \delta q$, so that the logarithmic jump at $r_{*}$, i.e. $\mathbb{C}_{+}=\left.\frac{r X_{+}^{\prime}(r)}{X_{+}(r)}\right|_{r_{*}-x}$ is readily computed:

$$
\mathbb{C}_{+}=m-\frac{2(1+m) / m_{*}}{\frac{m}{m_{*}}\left(\frac{r_{*}}{r_{0}}\right)^{2 m+2}-\left(\frac{r_{*}}{r_{0}}\right)^{2 m}+\frac{1}{m_{*}}}+\frac{2(m+1)}{1+n \delta q} .
$$

The lower $m_{-}=m-1$ mode $\left(X_{-}=X_{m-1}\right)$ has a resonance at $r_{s}=r_{*} \sqrt{\frac{m-1}{m_{*}}}$. Similarly to the upper mode, the solution is:

$$
X_{-}\left(r \gtrless r_{s}\right) \propto \frac{1}{k_{\|, m_{-}}}\left[\left(r / r_{s}\right)^{m-2}+B_{\gtrless}\left(r / r_{s}\right)^{-m}\right],
$$

where $B_{<}=\left(r_{s} / r_{0}\right)^{2-2 m} /\left[m-2-m_{*}\left(\frac{r_{0}}{r_{*}}\right)^{2}\right]$. The logarithmic jump of the lower mode $\mathbb{C}_{-}=\left.\frac{r X_{-}^{\prime}(r)}{X_{-}(r)}\right|_{r_{*}-x}$ is therefore given by:

$$
\mathbb{C}_{-}=-m+\frac{2(m-1)}{1+B_{>}\left(\frac{r_{*}}{r_{s}}\right)^{2-2 m}}-\frac{2(m-1)}{1-n \delta q}
$$

The quantity $B_{>}$is determined by matching the asymptotics of $X_{-}$when $r_{s}$ is approached with the solution obtained in a neighbourhood of the resonance where inertial effects and residual coupling terms could be retained (as generally speaking the main harmonic is not exactly vanishing for $r<r_{*}$ ). However in our approximation we assume that $\hat{\gamma} \ll 1$ and that coupling effects are negligible for $r<r_{*}$ so that we approximate $B_{>}=-1$ requiring that the mode is finite at its own resonant surface.

\section{PEDESTAL LOW-SHEAR REGION}

In this region of width $a-r_{*}=\Delta$ with $\Delta / a \sim \varepsilon \ll 1$, large pressure gradients drive large edge bootstrap current contributions which in turn flatten the safety factor. We adopt step-like profiles which mimic the abrupt decrease of pressure, density and rotation in this region $[3,4]$ :

$$
p_{0} / p_{*} \sim \rho_{0} / \bar{\rho} \sim \Omega / \Omega_{*}=\Theta\left(r_{p}-r\right)
$$

where $\Theta(x)$ is the Heaviside step function of argument $x$ while $p_{*}$ and $\Omega_{*}$ are the values of plasma pressure and toroidal rotation at $r_{*}$. The mass density is assumed constant over $0<r<r_{*}$. Since profiles for $p_{0}$ and $\rho_{0}$ are step-like, the gradients are vanishing everywhere except at one point, so that we can conveniently employ Eqs. (2) and (3).

First we integrate (3) across $r_{p}$ giving $\llbracket\left(r^{2 \pm m} X_{ \pm}\right)^{\prime} \rrbracket_{r_{p}}=0$. Integrating once (3) and inserting the result into (2) yields (1$\left.1 / q^{2} \approx 1\right)$ :

$$
\begin{gathered}
{\left[r^{3} Q X_{m}^{\prime}\right]^{\prime}+r\left[\left(1-m^{2}\right) Q+r\left(\frac{A_{2}}{n^{2}}\right)^{\prime}-\frac{\tilde{\alpha} r}{R_{0}}\right] X_{m}} \\
+\frac{\tilde{\alpha}}{2}\left[\frac{r^{1+m} L_{+}}{1+m}+\frac{r^{1-m} L_{-}}{1-m}\right]=0 \\
\left(r^{2 \pm m} X_{m \pm 1}\right)^{\prime}=r^{1 \pm 2 m} L_{ \pm}+\frac{1 \pm m}{2} \tilde{\alpha} r^{1 \pm m} X_{m}
\end{gathered}
$$


Employing (8) it follows that $A_{1} \simeq-\Theta\left(r_{p}-r\right)\left(\omega_{D *} / \omega_{A}\right)^{2}(1+$ $\left.2 q^{2}\right), A_{2} \simeq-\Theta\left(r_{p}-r\right) \omega\left[\omega\left(1+2 q^{2}\right)+2 n \Omega_{*}\right] / \omega_{A}^{2}$ and $\tilde{p}_{0}=$ $\tilde{p}_{*} \Theta\left(r_{p}-r\right)$ with $\tilde{p}_{*}=p_{*}+B_{0}^{2} / 2\left(\Omega_{*} / \omega_{A}\right)^{2}$ and $\omega_{D *}=\omega+n \Omega_{*}$. Here we have $q=m / n-\delta q$.

Because of the nature of the $\delta$-like singularity in $\tilde{\alpha}$ and $A_{2}^{\prime}$ in Eqs. (9) and (10), we expect a discontinuity of $X_{ \pm}, X_{ \pm}^{\prime}$ and $X_{m}^{\prime}$ at $r_{p}$ while $X_{m}$ remains continuous. Using the profiles given in (8), the equation for $X_{m}$ is simplified as follows:

$$
\left[r^{3} X_{m}^{\prime}\right]^{\prime}+r\left(1-m^{2}\right) X_{m}=0
$$

which holds both for $r<r_{p}$ and $r>r_{p}$ with $r_{*}<r<a$. The solution of the equation above must be matched with the solution in the inner and vacuum regions requiring continuity at $r_{p}$. Thus we impose the constraint:

$$
X_{m}\left(r_{*}\right)=X_{m}(a)=0 .
$$

Hence the solution of (11), continuous at $r_{p}$, and fulfilling the boundary conditions at $r_{*}$ and $a$, is:

$$
X_{m}=X_{0} \times \begin{cases}\frac{\left(\frac{r}{r_{*}}\right)^{m-1}-\left(\frac{r}{r_{*}}\right)^{-m-1}}{\left(\frac{r_{p}}{r_{*}}\right)^{m-1}-\left(\frac{r_{p}}{r_{*}}\right)^{-m-1},} & r<r_{p}, \\ \frac{\left(\frac{r}{a}\right)^{m-1}-\left(\frac{r}{a}\right)^{-m-1}}{\left(\frac{r_{p}}{a}\right)^{m-1}-\left(\frac{r_{p}}{a}\right)^{-m-1}}, & r>r_{p} .\end{cases}
$$

The sideband harmonics must also be supplied with appropriate boundary conditions. These are obtained by integrating (3) across $r_{*}$ and $a$ yielding:

$$
\begin{gathered}
\left.\mathbb{C}_{ \pm} \equiv \frac{r X_{ \pm}^{\prime}(r)}{X_{ \pm}(r)}\right|_{r_{*}-x}=\left.\frac{r X_{ \pm}^{\prime}(r)}{X_{ \pm}(r)}\right|_{r_{*}+x}, \\
\left.\mathbb{B}_{ \pm} \equiv \frac{r X_{ \pm}^{\prime}(r)}{X_{ \pm}(r)}\right|_{a+x}=\left.\frac{r X_{ \pm}^{\prime}(r)}{X_{ \pm}(r)}\right|_{a-x}
\end{gathered}
$$

Equation (14) is evaluated by means of (6) and (7) while (15) is obtained from (4) (see Ref. [28]).

Evaluating (10) at $r_{*}$ and $a$ gives respectively $X_{ \pm}\left(r_{*}\right)=$ $r_{*}^{ \pm m} L_{ \pm} /\left(2 \pm m+\mathbb{C}_{ \pm}\right)$and $X_{ \pm}(a)=a^{ \pm m} L_{ \pm} /\left(2 \pm m+\mathbb{B}_{ \pm}\right)$having used (12). Integration of Eq. (10) from $r_{*}$ to $a$ finally gives $\frac{r_{p}^{ \pm m} L_{ \pm}}{1 \pm m}=X_{0} \frac{\hat{\beta}}{\varepsilon_{p}} \Lambda^{( \pm)}$, where $\hat{\beta}=2 \tilde{p}_{*} q^{2} / B_{0}^{2}, \varepsilon_{p}=r_{p} / R_{0}$ and [23]:

$\Lambda^{( \pm)}=\frac{\left(r_{p} / r_{*}\right)^{2 \pm 2 m}(1 \pm m)\left[2 \pm m+\mathbb{C}_{ \pm}\right]\left[2 \pm m+\mathbb{B}_{ \pm}\right]}{\left(\mathbb{C}_{ \pm} \mp m\right)\left[2 \pm m+\mathbb{B}_{ \pm}\right]-\left(\mathbb{B}_{ \pm} \mp m\right)\left[2 \pm m+\mathbb{C}_{ \pm}\right]\left(\frac{a}{r_{*}}\right)^{2 \pm 2 m}}$

Let us define the following quantities:

$$
\begin{gathered}
g_{-}=\left.\frac{r X_{m}^{\prime}}{X_{m}}\right|_{r_{p}^{-}}=\frac{m-1+(m+1)\left(r_{*} / r_{p}\right)^{2 m}}{1-\left(r_{*} / r_{p}\right)^{2 m}}>0, \\
g_{+}=\left.\frac{r X_{m}^{\prime}}{X_{m}}\right|_{r_{p}^{+}}=\frac{m-1+(m+1)\left(a / r_{p}\right)^{2 m}}{1-\left(a / r_{p}\right)^{2 m}}<0 .
\end{gathered}
$$

Following Ref. [25], the dispersion relation is obtained by integrating (9) across $r_{p}$. Using the expressions for $L_{ \pm}$and approximating $g_{-} \approx-g_{+} \approx 2 a / \Delta \gg 1$ with $m$ not too large, we obtain:

$$
\frac{\left(\gamma-i n \Omega_{*}\right)^{2}\left[1+2 q^{2}\right]}{2 n^{2} \omega_{A}^{2}}=\frac{\Delta}{4 a}\left[\frac{\hat{\beta}^{2}}{2 \varepsilon_{p}^{2}}\left(\Lambda^{(+)}+\Lambda^{(-)}\right)-\hat{\beta}\right]-\left(\frac{\delta q}{q}\right)^{2} .
$$

where $\Lambda^{( \pm)}$are given by (16). Note that no numerical integration for calculating the growth rate is required. We stress the point, already stated in the description of the physical model, that the theory holds under the assumption that $k_{\|, m}\left(r_{*}\right) \sim \delta q / q \ll 1$. Since $\delta q$ is divided by $q$ we may allow $\delta q$ to be moderately small.

With an ideally conducting wall the coefficients $\Lambda^{( \pm)}$do not depend on $\omega$ and toroidal rotation effects enter at leading order through a Doppler shift of the eigenfrequency (the $\beta$ modification due to centrifugal effects has a rather weak effect). It is found numerically that the rhs of (17) becomes negative when $b=a$ implying stability. For $b>a$, the growth rate increases with $n[11,15]$ suggesting that the linear phase is dominated by modes with moderately large $n$ (peeling-ballooning modes are considered stable since we assume to operate below the peeling-ballooning stability boundary). The growth rates computed from (17) for the ideally conducting wall case are shown in Fig. 2 in the limit $\Omega_{*}=0$ for the mode $m=4, n=1$. Having retained the $\delta q(=m / n-q)$ dependence in the coefficients $\mathbb{C}_{ \pm}$and $\mathbb{B}_{ \pm}$, the growth rate is not symmetric in $\delta q$, i.e. an upwards shift of the safety factor $(\delta q<0)$ leads to a more unstable situation than a downwards shift $(\delta q>0)[10,11]$. This gives different marginal stability boundaries depending on the sign of $\delta q$. We stress the point however that we shall keep $\delta q$ small enough in order to fulfil the the condition $X_{m}\left(r_{*}\right) \approx X_{m}(a) \approx 0$.

We simplify Eq. (17) by approximating $\mathbb{C}_{+} \approx 3 m+2$ and $\mathbb{C}_{-} \approx m / 6-1 / 4$ and neglecting $\delta q$ corrections in $\mathbb{B}_{ \pm}$. If Eq. (17) is solved for $\gamma$, we immediately see that $\operatorname{Im}(\gamma)=n \Omega_{*}$, while the rotation effects do not enter into $\operatorname{Re}(\gamma)$ (i.e. the growth rate). This indicates, as stated previously, that toroidal plasma rotation simply Doppler shifts the mode. Taking the limit $r_{p} \sim r_{*} \sim a$ for $\Delta=a-r_{*} \ll 1$ if $m$ is sufficiently large we get $\Lambda^{(+)} \approx 2 m\left[1-2(a / b)^{2 m}\right]$ and $\Lambda^{(-)} \approx m / 2$, yielding the following approximate instability criterion:

$$
\frac{m}{2}\left(\frac{\Delta}{a}\right) \frac{\hat{\beta}^{2}}{\varepsilon_{p}^{2}}\left[\frac{5}{8}-\left(\frac{a}{b}\right)^{2 m}\right]>\left(\frac{\delta q}{q}\right)^{2} .
$$

The increase of pressure gradients $(\hat{\beta} \uparrow)$ and the field line bending weakening $(\delta q \downarrow)$ drive the instability. In order to satisfy the infernal ordering (only neighbouring sidebands contribute) the width of the coupling (namely pedestal low-shear) region has to shrink when $m$ increases yielding $\Delta / a \propto 1 / m$ so that the stability boundary becomes independent of $m$. Having defined the current distribution (i.e. $q, \delta q, \Delta$ ), the equation above provides a criterion for $\hat{\beta}$. To give an example, with $q \sim 4, \delta q \approx 0.1, \varepsilon_{p}=1 / 3, b / a=1.3$ and $\Delta / a \approx 5 \times 10^{-2}$ (as in Fig. 2) the instability condition gives $\hat{\beta} \gtrsim 3.7 \%$.

\section{CONCLUSIONS}

The dispersion relation for edge located ideal infernal-like instabilities has been derived in $\mathrm{QH}$-like tokamak regimes with a simple class of profiles for pressure, mass density and equilibrium toroidal rotation. Fundamental for our analysis is the edge local flattening of the safety factor, local sharp 

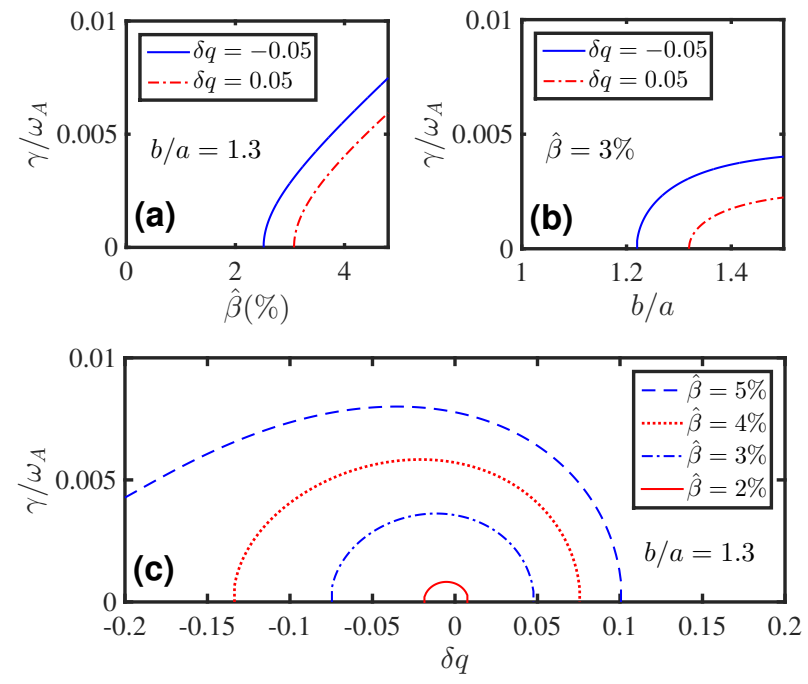

Figure 2. Growth rates computed from Eq. (17) for the the mode $m / n=4 / 1$ (ideal wall case) with $q=m / n-\delta q, \varepsilon=1 / 3, r_{0} / a=0.5$, $r_{*} / a=0.95\left(\Delta / a=5 \times 10^{-2}\right), \Omega_{*}=0$ wrt $\hat{\beta}(\mathrm{a})$, wall position (b) and $\delta q(\mathrm{c})$. The growth rate $\gamma$ is not symmetric in $\delta q$ because of the $\delta q$ dependence of the coefficients $\mathbb{C}_{ \pm}$and $\mathbb{B}_{ \pm}$. We stress the point that these results remain valid within the approximation $\delta q / q \ll 1$. pressure gradients, and the presence of a vacuum gap between plasma and the metallic wall all of which are relevant in the conditions of quiescent $\mathrm{H}$-mode regimes. It is shown that instability occurs when the safety factor is close to the rational value at the edge. Similarly to the standard infernal modes, and unlike the standard external kink mode, instability occurs even when $q_{a}>m / n$ providing that the pressure is large enough. Nevertheless, in keeping with external kink modes, the plasma is stable for all $\beta$ and $q_{a}$ if the perfectly conducting wall is placed on the plasma boundary. Although a simple limited geometry has been chosen, various features measured experimentally and modelled numerically for similar configurations have been retrieved. Indeed intriguing similarities between such perturbations and EHOs are found: the structure of the radial eigenfunction (cf. (13)) resembles closely experimental measurements [5], the rotation frequency of the mode scales as $n \Omega_{*}$ (with $\Omega_{*}$ the value of toroidal rotation at the pedestal top) with the growth rate (and presumably the saturated non-linear state) being independent of the sign of $\Omega_{*}$. A further extension of this work will be to include $\mathrm{X}$-point effects but this is beyond the scope of the present paper.

Fruitful discussions with Dr D. Dragoni are acknowledged.
[1] Burrell K H et al. 2002 Plasma Phys. Control. Fusion 44 A253

[2] Suttrop W et al. 2003 Plasma Phys. Control. Fusion 451399

[3] Burrell K H et al. 2001 Phys. Plasmas 82153

[4] Burrell K H et al. 2005 Phys. Plasmas 12056121

[5] Chen X et al. 2016 Nucl. Fusion 56076011

[6] Suttrop W et al. 2005 Nucl. Fusion 45721

[7] Solano E R et al. 2010 Phys. Rev. Lett. 104185003

[8] Connor J W et al. 1998 Phys. Plasmas 52687

[9] Medvedev S Y et al. 2006 Plasma Phys. Control. Fusion 48927

[10] Zheng L J et al. 2013 Phys. Plasmas 20012501

[11] Zheng L J et al. 2013 Nucl. Fusion 53063009

[12] Cooper W A et al. 2015 J. Plasma Phys. 81515810605

[13] Cooper W A et al. 2016 Plasma Phys. Control. Fusion 58 064002

[14] Cooper W A et al. 2016 Phys. Plasmas 23040701

[15] Liu F et al. 2015 Nucl. Fusion 55113002

[16] Bussac M N et al. 1975 Phys. Rev. Lett. 351638

[17] Hazeltine R D and Meiss J D 1992 Plasma Confinement (Red- wood City: Addison-Wesley Publishing Company)

[18] Turnbull A D and Troyon F 1989 Nucl. Fusion 291887

[19] Frieman E and Rotenberg M 1960 Rev. Mod. Phys. 32898

[20] Newcomb W 1960 Ann. Phys. 10232

[21] Mikhailovskii A B 1998 Instabilities in a Confined Plasma (Bristol: IOP)

[22] Hastie R J and Hender T C 1988 Nucl. Fusion 28585

[23] Gimblett C G et al. 1996 Phys. Plasmas 33369

[24] Wahlberg C and Graves J P 2011 38th EPS Conf. on Plasma Physics (European Physical Society, Strasbourg, France, paper no P5.089

[25] Wahlberg C et al. 2013 Plasma Phys. Control. Fusion 55 105004

[26] Ham C J et al. 2012 Plasma Phys. Control. Fusion 54025009

[27] Wahlberg C 2009 Plasma Phys. Control. Fusion 51085006

[28] Wesson J A 1978 Nucl. Fusion 1887

[29] Bernstein I B et al. 1958 Proc. R. Soc. Lond. A 24417

[30] Freidberg J P 1987 Ideal Magnetohydrodynamics (New York: Plenum Press) 